PROCEEDINGS OF THE

AMERICAN MATHEMATICAL SOCIETY

Volume 131, Number 4, Pages 1273-1282

S 0002-9939(02)06794-1

Article electronically published on November 20, 2002

\title{
ORDERED GROUP INVARIANTS FOR NONORIENTABLE ONE-DIMENSIONAL GENERALIZED SOLENOIDS
}

\author{
INHYEOP YI
}

(Communicated by Michael Handel)

\begin{abstract}
Let $f: X \rightarrow X$ be an edge-wrapping rule which presents a onedimensional generalized solenoid $\bar{X}$, and let $M$ be the adjacency matrix of $f$. When $X$ is a wedge of circles and $f$ leaves the unique branch point fixed, we show that the stationary dimension group of $M$ is an invariant of homeomorphism of $\bar{X}$ even if $\bar{X}$ is not orientable.
\end{abstract}

\section{Introduction}

This paper is part of a study of certain one-dimensional spaces which can be associated to invariant sets in dynamical systems. In recent developments, ordered group invariants in $C^{*}$-algebras and topological dynamics have been used to provide invariants of homeomorphisms of certain one-dimensional compact metrizable spaces $([1],[2], 3], 6]$, 9], [10], [11, [16]). We can interpret these results as follows: the first Cech cohomology group of the space is given a preorder, and the isomorphism class of the resulting preordered group becomes an invariant of the homeomorphism class of the space.

An important type of ordered group arising in this setting is the dimension group $\left(\Delta_{M}, \Delta_{M}^{+}\right)$defined by an $n \times n$ nonnegative integer matrix $M$. Here $\Delta_{M}$ is the direct limit group $\lim \mathbb{Z}^{n}=\left\{(\mathbf{v}, k) \mid \mathbf{v} \in \mathbb{Z}^{n}, k \in \mathbb{N}\right\} / \sim$, with $(\mathbf{v}, k) \sim\left(\mathbf{v}^{\prime}, k^{\prime}\right)$ if there exist $i, j \in \mathbb{N}$ such that $i+k=j+k^{\prime}$ and $M^{i} \mathbf{v}=M^{j} \mathbf{v}^{\prime}$. And the positive set is $\Delta_{M}^{+}=\left\{[(\mathbf{v}, k)] \mid M^{i} \mathbf{v} \geq 0\right.$ for some $\left.i \in \mathbb{N}\right\}$. The order structure gives rise to additional number-theoretic invariants $([1],[5])$. In the case that two nonnegative integer matrices $M_{1}$ and $M_{2}$ are primitive (the case relevant to this paper), there is a procedure to determine whether the dimension groups $\left(\Delta_{M_{1}}, \Delta_{M_{1}}^{+}\right)$and $\left(\Delta_{M_{2}}, \Delta_{M_{2}}^{+}\right)$ are isomorphic $([5])$.

One class of topological spaces studied here is the class of one-dimensional generalized solenoids (1-solenoids) introduced by R. F. Williams. The 1solenoids were employed to describe a theory of one-dimensional hyperbolic expanding attractors ([13, [14]). A 1-solenoid is a space homeomorphic to the inverse limit space $\bar{X}$ obtained from a map $f: X \rightarrow X$ where $X$ is a graph and $f$ satisfies certain axioms $([13],[15])$. The map $f$ has a transition matrix $M_{X, f}=M$

Received by the editors June 27, 2001.

1991 Mathematics Subject Classification. Primary 58F03, 58F12, 54H20.

Key words and phrases. One-dimensional generalized solenoid, orientable double cover, ordered group. 
where $M(i, j)$ is the number of times the $i^{\text {th }}$ edge of $X$ is covered by the $j^{\text {th }}$ edge. The presentation $(X, f)$ is elementary if $X$ is a wedge of circles. Every 1-solenoid has an elementary presentation ([14], [15]). If $(X, f)$ is an elementary presentation of a 1 -solenoid $\bar{X}$ and $M=M_{X, f}$ is the adjacency matrix of $f: X \rightarrow X$, then $\check{H}^{1}(\bar{X})$ is isomorphic to $\Delta_{M}([16])$. This invariant of the homeomorphism class of $\bar{X}$ was refined by Jacklitch $(\underline{3})$, who showed that if $(X, f)$ and $(Y, g)$ are elementary presentations of oriented 1-solenoids $\bar{X}$ and $\bar{Y}$, then the dimension groups $\left(\Delta_{M_{X, f}}, \Delta_{M_{X, f}}^{+}\right)$and $\left(\Delta_{M_{Y, g}}, \Delta_{M_{Y, g}}^{+}\right)$are isomorphic. We can define a positive set $\check{H}_{\oplus}^{1}$ for the winding order on $\check{H}^{1}$ for a large class of one-dimensional spaces, and explain the Jacklitch result as the computation of $\left(\check{H}^{1}, \check{H}_{\oplus}^{1}\right)$ for 1-solenoids ([16]). This can also be done by analyzing the direct limit order on $\check{H}^{1}$, and we can recast the arguments of [3] and [9] in this framework.

In this paper, we extend the Jacklitch result to the nonorientable case with the following proof scheme. Given a nonorientable solenoid $\bar{X}$, there is a canonical double covering $\bar{p}: \overline{\tilde{X}} \rightarrow \bar{X}$ where $\overline{\tilde{X}}$ is an orientable 1-solenoid ([17]). Then the quotient group $\check{H}^{1}(\overline{\tilde{X}}) / \bar{p}^{*}\left(\check{H}^{1}(\bar{X})\right)$ is a homeomorphism invariant of $\bar{X}$. When $(X, f)$ is an elementary presentation, the quotient group is isomorphic to $\Delta_{M_{X, f}}$, and when $(X, f)$ is an orientable elementary presentation, the quotient group with the canonical order structure is isomorphic to $\left(\Delta_{M_{X, f}}, \Delta_{M_{X, f}}^{+}\right)$. The assumption of an elementary presentation is necessary and natural. If $\bar{X}$ is orientable and elementary presented, then $\Delta_{M_{X, f}}$ is order isomorphic to $\check{H}^{1}(\overline{\tilde{X}})$; but this may fail if the presentation is not elementary ([16]). Likewise, if $\bar{X}$ is not orientable and $(X, f)$ is not an elementary presentation, then $\Delta_{M_{X, f}}$ need not be isomorphic to $\check{H}^{1}(\overline{\tilde{X}}) / \bar{p}^{*}\left(\check{H}^{1}(\bar{X})\right)$.

\section{ONE-DIMENSIONAL GENERALIZED SOLENOIDS AND ORDERED GROUPS}

We review the definitions of one-dimensional generalized solenoids of Williams and ordered groups which will be used in later sections. As general references for the notions of one-dimensional generalized solenoids and their ordered group invariants we refer to [13], [14], [15].

One-dimensional generalized solenoids. Let $X$ be a directed graph with vertex set $\mathcal{V}$ and edge set $\mathcal{E}$, and $f: X \rightarrow X$ a continuous map. We state some axioms which might be satisfied by $(X, f)$.

Axiom 0. (Indecomposability) $(X, f)$ is indecomposable.

Axiom 1. (Nonwandering) All points of $X$ are nonwandering under $f$.

Axiom 2. (Flattening) There is $k \geq 1$ such that for every $x \in X$ there is an open neighborhood $U$ of $x$ such that $f^{k}(U)$ is homeomorphic to $(-\epsilon, \epsilon)$.

Axiom 3. (Expansion) There is a metric $d$ compatible with the topology and positive constants $C$ and $\lambda$ with $\lambda>1$ such that for all $n>0$ and all points $x, y$ on a common edge of $X$, if $f^{n}$ maps the interval $[x, y]$ into an edge, then $d\left(f^{n} x, f^{n} y\right) \geq C \lambda^{n} d(x, y)$.

Axiom 4. (Nonfolding) $\left.f^{n}\right|_{X-\mathcal{V}}$ is locally one-to-one for every positive integer $n$. Axiom 5. (Markov) $f(\mathcal{V}) \subseteq \mathcal{V}$. 
Let $\bar{X}$ be the inverse limit space

$$
\bar{X}=X \stackrel{f}{\longleftarrow} X \stackrel{f}{\longleftarrow} \cdots=\left\{\left(x_{0}, x_{1}, x_{2}, \ldots\right) \in \prod_{0}^{\infty} X \mid f\left(x_{n+1}\right)=x_{n}\right\} .
$$

Suppose that $Y$ is a topological space. We call $Y$ a 1-dimensional generalized solenoid or 1-solenoid if there exists a directed graph $X$ and a continuous map $f: X \rightarrow X$ such that $(X, f)$ satisfies all six axioms and $\bar{X}$ is homeomorphic to $Y$. And $(X, f)$ is called a presentation of $Y$. If we can choose the direction of each edge in $X$ so that the connection map $f: X \rightarrow X$ is orientation preserving, then we call $(X, f)$ an oriented presentation, and $Y$ an orientable solenoid. We call a point $x \in X$ a non-branch point if $x$ has an open neighborhood which is homeomorphic to an open interval, and branch point otherwise. An elementary presentation $(X, f)$ of a 1-solenoid is such that $X$ is a wedge of circles and $f$ leaves the unique branch point of $X$ fixed.

Proposition 2.1 ([14, 5.2]). For every 1 -solenoid $\bar{X}$, there is an elementary presentation $(Y, g)$ such that $\bar{X}$ is homeomorphic to $\bar{Y}$.

Notation 2.2. Suppose that $(X, f)$ is a presentation of a 1-solenoid, and that $\mathcal{E}=\left\{e_{1}, \ldots, e_{n}\right\}$ is the edge set of the directed graph $X$. For each edge $e_{i} \in \mathcal{E}$, we can give $e_{i}$ the partition $\left\{I_{i, j}\right\}, 1 \leq j \leq l(i)$, such that

(1) the initial point of $I_{i, 1}$ is the initial point of $e_{i}$,

(2) the terminal point of $I_{i, j}$ is the initial point of $I_{i, j+1}$ for $1 \leq j<l(i)$,

(3) the terminal point of $I_{i, l(i)}$ is the terminal point of $e_{i}$,

(4) $\left.f\right|_{\operatorname{Int} I_{i, j}}$ is injective, and

(5) $f\left(I_{i, j}\right)=e_{i, j}^{s(i, j)}$ where $e_{i, j} \in \mathcal{E}, s(i, j)=1$ if the direction of $f\left(I_{i, j}\right)$ agrees with that of $e_{i, j}$, and $s(i, j)=-1$ if the direction of $f\left(I_{i, j}\right)$ is reverse to that of $e_{i, j}$.

By a path in $X$ we mean a finite sequence $e_{1}^{s(1)} \cdots e_{n}^{s(n)}$ of edges such that, for $1 \leq i<n, s(i)= \pm 1$ represents the direction of $e_{i}$ and the terminal vertex of $e_{i}^{s(i)}$ is the initial vertex of $e_{i+1}^{s(i+1)}$. Let $\mathcal{E}^{*}$ denote the path set of $X$. Then the wrapping rule $\check{f}: \mathcal{E} \rightarrow \mathcal{E}^{*}$ associated with $f$ is given by

$$
\check{f}: e_{i} \mapsto e_{i, 1}^{s(i, 1)} \cdots e_{i, l(i)}^{s(i, l(i))},
$$

and the adjacency matrix $M=M_{X, f}$ of $(\mathcal{E}, \breve{f})$ is given by

$$
M(i, k)=\#\left\{I_{i, j} \mid f\left(I_{i, j}\right)=e_{k}^{ \pm 1}\right\} .
$$

Definition $2.3([7, \S 2])$. A preordered group is a pair $\left(G, G_{+}\right)$where $G$ is an abelian group and the positive cone $G_{+}$is a submonoid of $G$ which generates $G$. We write $g_{1} \leq g_{2}$ if $g_{1}-g_{2} \in G_{+}$for $g_{1}, g_{2} \in G$. If $\left(G, G_{+}\right)$satisfies the additional condition that $G_{+} \cap-G_{+}=\{0\}$, then $\left(G, G_{+}\right)$is called an ordered group.

For an oriented 1-solenoid $\bar{X}$, we can define a positive cone $\check{H}_{\oplus}^{1}(\bar{X})$ for the winding order on the first Čech cohomology group so that $\left(\check{H}^{1}(\bar{X}), \check{H}_{\oplus}^{1}(\bar{X})\right)$ is an ordered group ([16]).

Proposition 2.4 ([16]). Suppose that $(X, f)$ is an oriented presentation of a 1 solenoid $\bar{X}$ with the adjacency matrix $M=M_{X, f}$.

(1) $\left(\check{H}^{1}(\bar{X}), \check{H}_{\oplus}^{1}(\bar{X})\right)$ is a quotient of $\left(\Delta_{M}, \Delta_{M}^{+}\right)$. 
(2) If $(X, f)$ is an elementary presentation, then $\left(\check{H}^{1}(\bar{X}), \check{H}_{\oplus}^{1}(\bar{X})\right)$ is isomorphic to $\left(\Delta_{M}, \Delta_{M}^{+}\right)$.

(3) Suppose that $\bar{Y}$ is an orientable 1-solenoid. If $h: \bar{X} \rightarrow \bar{Y}$ is a homeomorphism, then the induced map $h^{*}: \check{H}^{1}(\bar{X}) \rightarrow \check{H}^{1}(\bar{Y})$ defines an isomorphism of ordered groups $\left(\check{H}^{1}(\bar{X}), \check{H}_{\oplus}^{1}(\bar{X})\right)$ and $\left(\check{H}^{1}(\bar{Y}), \check{H}_{\oplus}^{1}(\bar{Y})\right)$.

\section{Orientable double COVER of 1-SOlenoids}

Suppose that $(X, f)$ is a presentation of a 1 -solenoid $\bar{X}$. We will make a new directed graph $\tilde{X}$, a lifting $\tilde{f}: \tilde{X} \rightarrow \tilde{X}$ of $f: X \rightarrow X$ and a double covering map $p: \tilde{X} \rightarrow X$ such that $f \circ p=p \circ \tilde{f}$. If $(X, f)$ is not orientable, then $(\tilde{X}, \tilde{f})$ is an orientable presentation of a 1 -solenoid $\overline{\tilde{X}}=\lim \tilde{X}$, and the induced map $\bar{p}: \overline{\tilde{X}} \rightarrow \bar{X}$

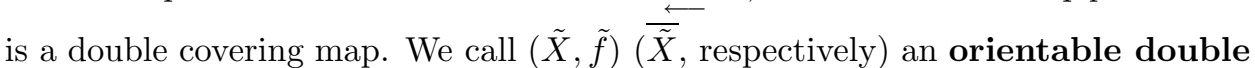
cover of $(X, f)(\bar{X}$, respectively). The existence of this orientable double cover is known $([8],[1])$. But we need to specify a concrete version of $(\tilde{X}, \tilde{f})$ for our computation.

Notation 3.1. We regard a directed edge $e$ of $X$ as a locally one-to-one map from $[0,1]$ to $e$ such that $e(0)$ is the initial point and $e(1)$ is the terminal point of $e$. So we can represent each point $x \in e$ as $e(t)$ (possibly $e(0)=e(1)$ ).

New graph $\tilde{X}$. Let $k \geq 1$ be an integer such that for every $x \in X$ there is an open neighborhood $U_{x}$ of $x$ such that $f^{k}\left(U_{x}\right)$ is homeomorphic to an open interval. Each edge $e_{i}$ of $X$ is the union of two subedges $e_{i, 1}=e_{i}\left(\left[0, \frac{1}{2}\right]\right)$ and $e_{i, 2}=e_{i}\left(\left[\frac{1}{2}, 1\right]\right)$. For a branch point $b$ of $X$, let $U_{b}$ be a neighborhood of $b$ given as above. Then we can choose $U_{b}$ to be small enough so that there exist two different subedges $e_{b}^{1}, e_{b}^{2} \in\left\{e_{i, j} \mid e_{i} \in \mathcal{E}\right.$ and $\left.j=1,2\right\}$ such that $f^{k}\left(U_{b}\right) \subset e_{b}^{1} \cup e_{b}^{2}$.

We can divide the set $\left\{e_{i, j} \mid b\right.$ is a boundary point of $\left.e_{i, j}\right\}$ into two disjoint sets

$$
U_{b}^{1}=\left\{e_{i, j} \mid f^{k}\left(e_{i, j} \cap U_{b}\right) \subset e_{b}^{1}\right\} \text { and } U_{b}^{2}=\left\{e_{i, j} \mid f^{k}\left(e_{i, j} \cap U_{b}\right) \subset e_{b}^{2}\right\} .
$$

As in [15. Appendix B], without loss of generality we can redraw the graph $X$ so that, for every branch point $b$, every subedge in $U_{b}^{1}$ comes to $b$ from the left or goes out from $b$ to the left, and every subedge in $U_{b}^{2}$ comes to $b$ from the right or goes out from $b$ to the right.

To make the double cover graph $\tilde{X}$, for each branch point $b$ of $X$ choose two points $b_{1}$ and $b_{2}$ which will be the fiber of $b$ in $\tilde{X}$. Draw copies of $U_{b}^{1}$ and $U_{b}^{2}$ at $b_{1}$ and $b_{2}$, and give the direction to each subedge in the liftings such that

(1) at $b_{1}$, every subedge contained in the copy of $U_{b}^{1}$ goes out from $b_{1}$ to the left and every subedge in the copy of $U_{b}^{2}$ comes in to $b_{1}$ from the right, and

(2) at $b_{2}$, every subedge contained in the copy of $U_{b}^{1}$ comes in to $b_{2}$ from the left and every subedge in the copy of $U_{b}^{2}$ goes out from $b_{2}$ to the right.

Now each subedge $e_{i, j}$ has two liftings in $\tilde{X}$, one of which has the same direction as $e_{i, j}$ and the other of which has the opposite direction. Let $e_{i, j}^{+}$be the lifting which has the same direction, and $e_{i, j}^{-}$the lifting with the opposite direction. Now connect $e_{i, 1}^{+}$to $e_{i, 2}^{+}$, and $e_{i, 1}^{-}$to $e_{i, 2}^{-}$so that

(1) for $E_{i, 1}=e_{i, 1}^{+} \cup e_{i, 2}^{+}, E_{i, 1}\left(\left[0, \frac{1}{2}\right]\right)=e_{i, 1}^{+}$and $E_{i, 1}\left(\left[\frac{1}{2}, 1\right]\right)=e_{i, 2}^{+}$, and

(2) for $E_{i, 2}=e_{i, 1}^{-} \cup e_{i, 2}^{-}, E_{i, 2}\left(\left[0, \frac{1}{2}\right]\right)=e_{i, 2}^{-}$and $E_{i, 2}\left(\left[\frac{1}{2}, 1\right]\right)=e_{i, 1}^{-}$. 
Then $E_{i, 1}, E_{i, 2}$ are liftings of $e_{i}$ in $\tilde{X}$ such that $E_{i, 1}$ corresponds to $e_{i}$ and that $E_{i, 2}$ corresponds to $e_{i}^{-1}$.

Example 3.2. Suppose that $X$ is Figure1. Then its double cover $\tilde{X}$ is given by Figure 2.

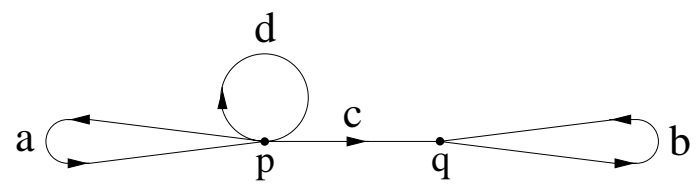

Figure 1. Directed graph $X$ for a nonorientable solenoid

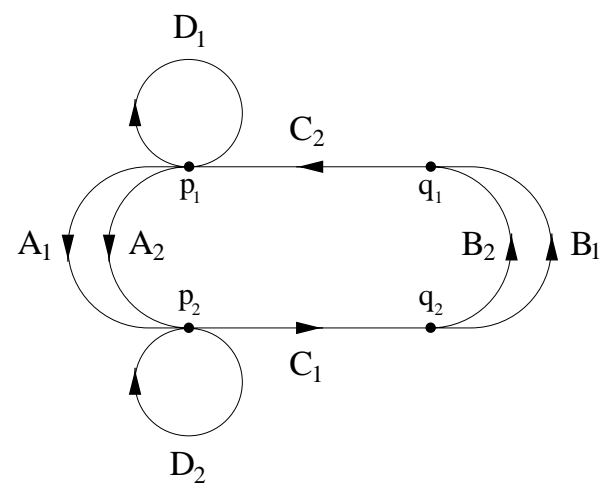

Figure 2. Double cover of $X$

Covering map. Define a map

$$
p: \tilde{X} \rightarrow X \text { by }\left\{\begin{array}{l}
E_{i, 1}(t) \mapsto e_{i}(t) \text { and } \\
E_{i, 2}(t) \mapsto e_{i}(1-t) .
\end{array}\right.
$$

Then $p$ is a 2-to-1 local homeomorphism. We call $\tilde{X}$ a covering space of $X$ and $p: \tilde{X} \rightarrow X$ a covering map.

The lifting $\tilde{f}: \tilde{X} \rightarrow \tilde{X}$. Suppose that $(X, f)$ is a nonorientable presentation, that $e_{i}:[0,1] \rightarrow X$ is an edge in $X$, that $E_{i, j}:[0,1] \rightarrow \tilde{X}, j=1,2$, are the liftings of $e_{i}$ in $\tilde{X}$, and $\check{f}\left(e_{i}\right)=e_{i(1)}^{s(1)} \cdots e_{i(n)}^{s(n)}$ where $s(j)$ represents the orientation. Then $p \circ E_{i, j}(0)=e_{i}(j-1)$ and $f \circ p \circ E_{i, j}(0)$ is the initial point of $e_{i(1)}^{s(1)}$ if $j=1$ and the terminal point of $e_{i(n)}^{s(n)}$ if $j=2$. Let

$$
x_{0}= \begin{cases}\text { the initial point of } E_{i(1), 1} & \text { if } j=1, s(1)=1, \\ \text { the initial point of } E_{i(1), 2} & \text { if } j=1, s(1)=-1, \\ \text { the initial point of } E_{i(n), 2} & \text { if } j=2, s(n)=1, \\ \text { the initial point of } E_{i(n), 1} & \text { if } j=2, s(n)=-1 .\end{cases}
$$

Then there exists a unique path $\Pi_{i, j}:[0,1] \rightarrow \tilde{X}$ such that $p \circ \Pi_{i, j}=f \circ p \circ E_{i, j}$ and $\Pi_{i, j}(0)=x_{0}$. Define $\tilde{f}: \tilde{X} \rightarrow \tilde{X}$ by $\left.\tilde{f}\right|_{E_{i, j}}=\Pi_{i, j} \circ E_{i, j}^{-1}$. 
Proposition 3.3. The map $\tilde{f}: \tilde{X} \rightarrow \tilde{X}$ is a well-defined orientation preserving continuous map such that $p \circ \tilde{f}=f \circ p$.

Proof. Since a nonbranch point is contained in a unique edge, if we show that $E_{i, j}(0)=E_{k, l}(1)=E_{m, n}(0)$ implies $\Pi_{i, j}(0)=\Pi_{k, l}(1)=\Pi_{m, n}(0)$, then $\tilde{f}$ is well defined and continuous. We only show that $E_{i, 1}(0)=E_{k, 1}(1)$ implies $\Pi_{i, 1}(0)=$ $\Pi_{k, 1}(1)$.

Suppose $\check{f}\left(e_{i}\right)=e_{i(1)}^{s(1)} \cdots e_{i(n)}^{s(n)}$ and $\check{f}\left(e_{k}\right)=e_{k(1)}^{t(1)} \cdots e_{k(m)}^{t(m)}$. Let $[0, a]$ and $[b, 1]$ be subintervals of $[0,1]$ such that $f \circ p \circ E_{i, 1}([0, a])=e_{i(1)}^{s(1)}\left(\left[0, \frac{1}{2}\right]\right)$ and $f \circ p \circ$ $E_{k, 1}([b, 1])=e_{k(m)}^{t(m)}\left(\left[\frac{1}{2}, 1\right]\right)$. Then, by the construction of $\tilde{X}$ and that $p$ is a local homeomorphism, we have $e_{i(1)}^{s(1)}\left(\left[0, \frac{1}{2}\right]\right) \in U_{b}^{1}$ if and only if $e_{k(m)}^{t(m)}\left(\left[\frac{1}{2}, 1\right]\right) \in U_{b}^{2}$ where $b=f \circ p \circ E_{i, 1}(0)$. If $\Pi_{i, 1}(0) \neq \Pi_{k, 1}(1)$, then the direction $\Pi_{i, 1}([0, a])$ that goes out from $\Pi_{i, 1}(0)$ and the direction $E_{k, 1}([b, 1])$ that comes to $\Pi_{k, 1}(1)$ are the same. Hence $p \circ \Pi_{i, 1}([0, a]) \in U_{b}^{1}$ if and only if $E_{k, 1}([b, 1]) \in U_{b}^{1}$, a contradiction. Therefore $\Pi_{i, 1}(0)=\Pi_{k, 1}(1)$, and $\tilde{f}$ is well defined. That $\tilde{f}: \tilde{X} \rightarrow \tilde{X}$ is a continuous map and that $p$ and $\tilde{f}$ satisfy $p \circ \tilde{f}=f \circ p$ are obvious.

Suppose that $[a, b] \subset[0,1]$ is an interval such that $\left.f \circ p \circ E_{i, j}\right|_{[a, b]}$ is a local homeomorphism and $f \circ p \circ E_{i, j}([a, b])=e_{k}^{-1}$. Then $p \circ \Pi_{i, j}([a, b])=f \circ p \circ$ $E_{i, j}([a, b])=e_{k}^{-1}$ implies $\Pi_{i, j}([a, b])=E_{k, 2}$, and $p \circ \Pi_{i, j}=f \circ p \circ E_{i, j}$ is a local homeomorphism on $[a, b]$ with $\Pi_{i, j}(a)=E_{k, 2}(0)$ and $\Pi_{i, j}(b)=E_{k, 2}(1)$. Therefore $\tilde{f}$ is orientation preserving.

Example 3.4. Suppose that $X$ and $\tilde{X}$ are as in Example 3.2 If $f: X \rightarrow X$ is given by

$$
a \mapsto c^{-1} d a c, b \mapsto c b c^{-1}, c \mapsto b c^{-1} a \text {, and } d \mapsto c^{-1} a c b^{-1},
$$

then $(X, f)$ is a presentation of a nonorientable solenoid and the lifting $\tilde{f}: \tilde{X} \rightarrow \tilde{X}$ is given by

$$
\begin{aligned}
& A_{1} \mapsto C_{2} D_{1} A_{1} C_{1}, B_{1} \mapsto C_{1} B_{1} C_{2}, C_{1} \mapsto B_{1} C_{2} A_{1}, D_{1} \mapsto C_{2} A_{1} C_{1} B_{2} \text { and } \\
& A_{2} \mapsto C_{2} A_{2} D_{2} C_{1}, B_{2} \mapsto C_{1} B_{2} C_{2}, C_{2} \mapsto A_{2} C_{1} B_{2}, D_{2} \mapsto B_{1} C_{2} A_{2} C_{1} .
\end{aligned}
$$

Lemma 3.5. Suppose that $(X, f)$ is a presentation of a nonorientable 1-solenoid with the edge set $\mathcal{E}$. Then for all $e_{1}, e_{2} \in \mathcal{E}$, there is a positive integer $n=n\left(e_{1}, e_{2}\right)$ such that $e_{1}, e_{1}^{-1} \in \check{f}^{n}\left(e_{2}\right)$ for every positive integer $n \geq n\left(e_{1}, e_{2}\right)$.

Proof. If there exists an edge $e \in \mathcal{E}$ such that $e$ and $e^{-1}$ are factors of $\check{f}^{n}(a)$ for some positive integer $n$ and an edge $a \in \mathcal{E}$, then by Theorem 1.2 of [14 for all $e_{1}, e_{2} \in \mathcal{E}, e_{1}, e_{1}^{-1} \in \check{f}^{n}\left(e_{2}\right)$ for some positive integer $n$. So we need to show the existence of such edges $e$ and $a$.

Assume that, for any two edges $a$ and $b$, only one of $b$ or $b^{-1}$ is a factor of $\check{f}^{n}(a)$ for every positive integer $n \geq n(a, b)$. Then the edge set $\mathcal{E}$ is divided into two disjoint sets $\mathcal{P}$ and $\mathcal{N}$ such that for any $a_{1}, a_{2} \in \mathcal{P}$ and $b_{1}, b_{2} \in \mathcal{N}, a_{2}$ and $b_{2}^{-1}$ are factors of $\check{f}^{n}\left(a_{1}\right)$ and $a_{2}^{-1}$ and $b_{2}$ are factors of $\check{f}^{n}\left(b_{1}\right)$ for every positive integer $n$. But if we give opposite direction to each edge $b \in \mathcal{N}$ so that $b^{-1} \in \mathcal{E}$, then the connection map $f: X \rightarrow X$ is orientable, which is a contradiction. Therefore $b$ and $b^{-1}$ are factors of $f^{n}(a)$ for all edges $a, b \in \mathcal{E}$ and every positive integer $n \geq n(a, b)$. 
Proposition 3.6. Suppose that $(X, f)$ is a presentation of a 1-solenoid. Then $(\tilde{X}, \tilde{f})$ is an orientable double cover of $(X, f)$.

(1) If $(X, f)$ is orientable, then $(\tilde{X}, \tilde{f})$ is the disjoint union of orientable presentations $\left(X_{1}, f_{1}\right)$ and $\left(X_{2}, f_{2}\right)$ of 1-solenoids such that $\left.p\right|_{X_{1}}$ is an orientation preserving homeomorphism, $\left.p\right|_{X_{2}}$ is an orientation reversing homeomorphism, and $\left(X_{i}, f_{i}\right)$ is topologically conjugate to $(X, f), i=1,2$.

(2) If $(X, f)$ is nonorientable, then $(\tilde{X}, \tilde{f})$ is an orientable presentation of a 1-solenoid.

Proof. (1) follows from Lemma 1.5 of [8] and the constructions of $(\tilde{X}, \tilde{f})$ and $p: \tilde{X} \rightarrow X$.

(2) The Expansion and Markov Axioms come from the construction of $(\tilde{X}, \tilde{f})$. The Indecomposability and Nonwandering Axioms are derived from Lemma 3.5.

Since $p$ is a local homeomorphism with $p(\tilde{X} \backslash \tilde{V})=X \backslash \mathcal{V}$ and $\left.f\right|_{X \backslash \mathcal{V}}$ is a local homeomorphism, $\left.\tilde{f}\right|_{\tilde{X} \backslash \tilde{V}}$ is a local homeomorphism, and $\tilde{f}$ satisfies the Nonfolding Axiom.

Suppose that $b$ is a branch point of $\tilde{X}$ and that $U$ is an open neighborhood of $p(b)$ such that $f^{k}(U)$ is an interval for some integer $k \geq 1$. Then there exists an open neighborhood $V$ of $b$ such that $\left.p\right|_{V}: V \rightarrow U$ is a homeomorphism. Since $p \circ \tilde{f}^{k}(V)=f^{k} \circ p(V)=f^{k}(U)$ is an interval and $p$ is a local homeomorphism, $\tilde{f}^{k}(V)$ is an interval. So $\tilde{f}$ satisfies the Flattening Axiom. Therefore $(\tilde{X}, \tilde{f})$ is an orientable presentation of a 1 -solenoid.

Proposition $3.7([8,1.6])$. Suppose that $\bar{X}$ and $\bar{Y}$ are 1-solenoids with orientable double covers $\overline{\tilde{X}}$ and $\overline{\tilde{Y}}$, respectively. Then a homeomorphism $h: \bar{X} \rightarrow \bar{Y}$ lifts to an orientation preserving homeomorphism $\tilde{h}: \overline{\tilde{X}} \rightarrow \overline{\tilde{Y}}$.

Sketch of Proof. We consider an arc component $\ell$ of a 1 -solenoid as a locally oneto-one map from $\mathbb{R}$ to $\ell$ so that each point $x \in \ell$ is represented as $\ell(t)$ and that $\left\{\ell_{1}(t), \ell_{2}(-t)\right\}$ in its orientable cover is a fiber of $\ell(t)$.

Let $\ell$ be an arc component of $\bar{X}$ and let $\ell_{1}$ and $\ell_{2}$ be liftings of $\ell$ in $\bar{X}$ such that $\ell_{1}$ has the same direction as $\ell$, and $\ell_{2}$ has the opposite direction. Suppose $h(\ell)=\ell^{\prime}$. Then $\ell^{\prime}$ is an arc component of $\bar{Y}$, and we give the direction to $\ell^{\prime}$ so that $\left.h\right|_{\ell}$ is orientation preserving. Let $\ell_{1}^{\prime}$ and $\ell_{2}^{\prime}$ be liftings of $\ell^{\prime}$ in $\bar{Y}$ such that $\ell_{1}^{\prime}$ has the same direction as $\ell^{\prime}$, and $\ell_{2}^{\prime}$ has the opposite direction. Define $\tilde{h}: \bar{X} \rightarrow \bar{Y}$ by $\ell_{i}(t) \mapsto \ell_{i}^{\prime}(t)$. Then $\tilde{h}$ is a well-defined homeomorphism satisfying $p_{Y} \circ \tilde{h}=h \circ p_{X}$.

Invariants for orientable double covers. Suppose that $\bar{X}$ is a 1-solenoid with a presentation $(X, f)$. Let $X_{k}=X$ be the $k$ th coordinate space of $\bar{X}$, and $\mathcal{E}_{k}$ the set of directed edges in $X_{k}$. Let $C\left(\mathcal{E}_{k}, \mathbb{Z}\right)$ denote the set of integer-valued functions on $\mathcal{E}_{k}$. If $e$ is an edge in $\mathcal{E}_{k+1}$ and $f: X_{k+1} \rightarrow X_{k}$ is the connection map, then $f(e)$ is a path $e_{1}^{s(1)} \cdots e_{n}^{s(n)}$ in $X_{k}$ where $e_{i}$ is an element of $\mathcal{E}_{k}$ and $s(i)$ is 1 or -1 according to whether the orientation is preserved or reversed. Hence $f$ induces a map

$$
f^{*}: C\left(\mathcal{E}_{k}, \mathbb{Z}\right) \rightarrow C\left(\mathcal{E}_{k+1}, \mathbb{Z}\right) \text { defined by } g \mapsto g \circ f
$$

where $g \circ f(e)=\sum_{i=1}^{n} s(i) g\left(e_{i}\right)$ such that $f(e)=e_{1}^{s(1)} \cdots e_{n}^{s(n)}$ in $X_{k}$. 
Remark 3.8. By Proposition 2.1] a 1-solenoid $\bar{X}$ is homeomorphic to an elementary presented 1-solenoid. So without loss of generality we can assume that $\bar{X}$ is elementary presented.

Suppose that $(X, f)$ is an elementary presentation of a nonorientable 1-solenoid with the edge set $\mathcal{E}$ and that $(\tilde{X}, \tilde{f})$ is its orientable double cover with the edge set $\tilde{\mathcal{E}}$ and the covering map $p: \tilde{X} \rightarrow X$. Let $M$ be the $n \times n$ adjacency matrix of $(\mathcal{E}, \tilde{f})$, and $\tilde{M}$ the $2 n \times 2 n$ adjacency matrix of $(\tilde{\mathcal{E}}, \tilde{\tilde{f}})$.

Proposition 3.9. Let $M$ and $\tilde{M}$ be given as above. Then there are two $n \times n$ nonnegative integer matrices $M_{1}$ and $M_{2}$ such that

$$
M=M_{1}+M_{2}, \check{H}^{1}(\bar{X})=\lim _{\longrightarrow} C\left(\mathcal{E}_{k}, \mathbb{Z}\right) \cong \Delta_{M_{1}-M_{2}}, \text { and } \tilde{M}=\left(\begin{array}{ll}
M_{1} & M_{2} \\
M_{2} & M_{1}
\end{array}\right) .
$$

Proof. Given presentation $(X, f)$, let $\left\{I_{i, j}\right\}$ be the partition of edge $e_{i}$ given in Notation 2.2. If $M_{1}$ and $M_{2}$ are the matrices given by

$$
M_{1}(i, k)=\#\left\{I_{i, j} \mid f\left(I_{i, j}\right)=e_{k}\right\} \text { and } M_{2}(i, k)=\#\left\{I_{i, j} \mid f\left(I_{i, j}\right)=e_{k}^{-1}\right\},
$$

then we have $M=M_{1}+M_{2}$.

Let $X_{k}$ be the $k$ th coordinate space of $\bar{X}$ and $g \in C\left(\mathcal{E}_{k}, \mathbb{Z}\right)$. Let $f^{*}: C\left(\mathcal{E}_{k}, \mathbb{Z}\right) \rightarrow$ $C\left(\mathcal{E}_{k+1}, \mathbb{Z}\right)$ be the induced homomorphism from $f: X_{k+1} \rightarrow X_{k}$. For edges $e_{i} \in$ $\mathcal{E}_{k+1}$ and $e_{j} \in \mathcal{E}_{k}$, if $f\left(e_{i}\right)$ covers $e_{j} n_{i, j}$-times in an orientation preserving way and $m_{i, j}$-times in an orientation reversing way, then

$$
g \circ f\left(e_{i}\right)=\sum\left(n_{i, j}-m_{i, j}\right) g\left(e_{j}\right) .
$$

It is not difficult to see that the matrix $\left(n_{i, j}\right)$ is equal to $M_{1}$ and that $\left(m_{i, j}\right)$ is equal to $M_{2}$. Hence $f^{*}: C\left(\mathcal{E}_{k}, \mathbb{Z}\right) \rightarrow C\left(\mathcal{E}_{k+1}, \mathbb{Z}\right)$ is denoted by the matrix $M_{1}-M_{2}$, and

$$
\check{H}^{1}(\bar{X})=\underset{f^{*}}{\lim } C\left(\mathcal{E}_{k}, \mathbb{Z}\right) \cong \underset{M_{1}-M_{2}}{\lim } \mathbb{Z}^{n}=\Delta_{M_{1}-M_{2}} .
$$

That $\tilde{M}=\left(\begin{array}{ll}M_{1} & M_{2} \\ M_{2} & M_{1}\end{array}\right)$ is an immediate conclusion from the construction of $(\tilde{X}, \tilde{f})$.

Then we have the following commuting diagram:

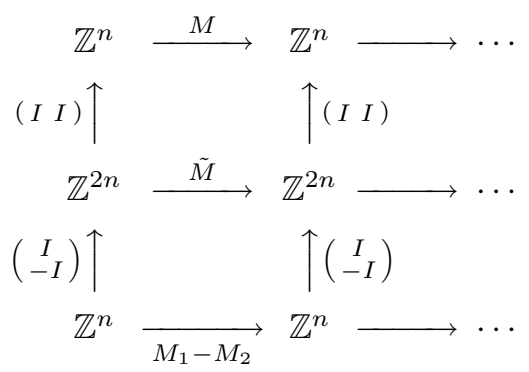

It is not difficult to see from the diagram that there is a well-defined homomorphism $p_{D}^{*}: \Delta_{M_{1}-M_{2}} \rightarrow \Delta_{\tilde{M}}$ induced by $\left(\begin{array}{c}I \\ -I\end{array}\right): \mathbb{Z}^{n} \rightarrow \mathbb{Z}^{2 n}$ such that $\Delta_{M}$ is isomorphic to $\Delta_{\tilde{M}} / p_{D}^{*}\left(\Delta_{M_{1}-M_{2}}\right)$. Remark that $\Delta_{\tilde{M}} / p_{D}^{*}\left(\Delta_{M_{1}-M_{2}}\right)$ is a preordered group with a positive cone $\Delta_{\tilde{M}}^{+} / p_{D}^{*}\left(\Delta_{M_{1}-M_{2}}\right)$. Then we have the following lemma.

Lemma 3.10. $\left(\Delta_{\tilde{M}} / p_{D}^{*}\left(\Delta_{M_{1}-M_{2}}\right), \Delta_{\tilde{M}}^{+} / p_{D}^{*}\left(\Delta_{M_{1}-M_{2}}\right)\right) \cong\left(\Delta_{M}, \Delta_{M}^{+}\right)$. 
Recall by Proposition 2.4 that there is an order preserving quotient map

$$
q:\left(\Delta_{\tilde{M}}, \Delta_{\tilde{M}}^{+}\right) \rightarrow\left(\check{H}^{1}(\overline{\tilde{X}}), \check{H}_{\oplus}^{1}(\overline{\tilde{X}})\right) .
$$

Suppose that $\tilde{X}_{k}=\tilde{X}$ is the $k$ th coordinate of $\overline{\tilde{X}}$, that $\pi_{k}: \overline{\tilde{X}} \rightarrow \tilde{X}_{k}$ is the projection map, and that $\tilde{\mathcal{E}}_{k}$ is the set of directed edges in $\tilde{X}_{k}$. By a path in $\tilde{X}$ we mean a finite sequence $e_{1}^{s(1)} \cdots e_{n}^{s(n)}$ of edges such that, for $1 \leq i<n, s(i)= \pm 1$ represents the direction of $e_{i}$ and the terminal vertex of $e_{i}^{s(i)}$ is the initial vertex of $e_{i+1}^{s(i+1)}$. We write $e^{s} \in \wp$ if $\wp$ is a path and $e$ is an edge such that $e^{s}$ is a factor of $\wp$. A cycle is a path $e_{1}^{s(1)} \ldots e_{n}^{s(n)}$ such that the terminal vertex of $e_{n}^{s(n)}$ is the initial vertex of $e_{1}^{s(1)}$. We say that a function $g$ in $C\left(\tilde{\mathcal{E}}_{k}, \mathbb{Z}\right)$ is zero on cycles if for every cycle $e_{1}^{s(1)} \cdots e_{n}^{s(n)}$ the sum $\sum s(i) g\left(e_{i}\right)$ vanishes.

Lemma 3.11 ([4], [16]). Suppose that $\pi_{k}: \bar{X} \rightarrow \tilde{X}_{k}$ is the projection map. Then the kernel of $q: \Delta_{\tilde{M}} \rightarrow \check{H}^{1}(\overline{\tilde{X}})$ is generated by $\left\{\pi_{k} \circ g \mid g\right.$ is zero on cycles in $\left.\tilde{X}_{k}\right\}$.

Then it is not difficult to verify that $q \circ p_{D}^{*}: \check{H}^{1}(\bar{X}) \rightarrow \check{H}^{1}(\overline{\tilde{X}})$ is the same as $\bar{p}^{*}: \check{H}^{1}(\bar{X}) \rightarrow \check{H}^{1}(\overline{\tilde{X}})$ induced by the double covering map $\bar{p}: \overline{\tilde{X}} \rightarrow \bar{X}$. Since $\operatorname{Ker}(q) \subset p_{D}^{*}\left(\check{H}^{1}(\bar{X})\right)$ from the above commuting diagram and Lemma 3.11 we have from Lemma 3.10 that $\check{H}^{1}(\overline{\tilde{X}}) / q \circ p_{D}^{*}\left(\check{H}^{1}(\bar{X})\right)$ is order isomorphic to $\Delta_{M}$. Therefore we have the following proposition.

Proposition 3.12. $\left(\check{H}^{1}(\overline{\tilde{X}}) / \bar{p}^{*}\left(\check{H}^{1}(\bar{X})\right), \check{H}_{\oplus}^{1}(\overline{\tilde{X}}) / \bar{p}^{*}\left(\check{H}^{1}(\bar{X})\right)\right) \cong\left(\Delta_{M}, \Delta_{M}^{+}\right)$.

\section{ACKNOWLEDGMENT}

I am grateful to Dr. M. Boyle for helpful discussions and encouragement, to Dr. R. Fokkink for sending me a copy of his unpublished Ph.D. thesis, and to the referee for useful suggestions.

\section{REFERENCES}

1. M. Barge and B. Diamond, Homeomorphisms of inverse limit spaces of one-dimensional maps, Fund. Math., 146 (1995), 509-537. MR 96b:54048

2. M. Barge and B. Diamond, A complete invariant for the topology of substitution tiling spaces, Ergodic Theory Dynam. Systems, 21 (2001), 1333-1358.

3. M. Barge, J. Jacklitch, and G. Vago, Homeomorphisms of One-dimensional Inverse Limits with Applications to Substitution Tilings, Unstable Manifolds, and Tent Maps, Geometry and Topology in Dynamics, Contemp. Math. 246 (1999), Amer. Math. Soc., 1-15. MR 2000j:37016

4. M. Boyle and D. Handelman, Orbit equivalence, flow equivalence and ordered cohomology, Israel J. Math., 95 (1996), 169-210. MR 98a:46082

5. O. Bratteli, P. Jorgensen, K. H. Kim and F. Rouch, Decidability of the isomorphism problem for stationary AF-algebras and the associated ordered simple dimension groups, Ergodic Theory Dynam. Systems, 21 (2001), 1625-1655. MR 2002h:46088

6. F. Durand, B. Host and C. Skau, Substitution dynamical systems, Bratteli diagrams and dimension groups, Ergodic Theory Dynam. Systems, 19 (1999), 953-993. MR 2001i:46062

7. E. Effros, Dimensions and $C^{*}$-algebras, CBMS Regional Conf. Ser. in Math. 46, Amer. Math. Soc., 1981. MR 84k:46042

8. R. Fokkink, The structure of trajectories, Ph.D. Thesis, Technische Universiteit te Delft, 1991.

9. A. Forrest, Cohomology of ordered Bratteli diagrams, To appear in Pacific J. Math.

10. T. Giordano, I. Putnam and C. Skau, Topological orbit equivalence and $C^{*}$-crossed products, J. Reine Angew. Math., 469 (1995), 41-111. MR 97g:46085 
11. R. Herman, I. Putnam and C. Skau, Ordered Bratteli diagram, dimension groups and topological dynamics, Intern. J. Math., 3 (1992), 827-864. MR 94f:46096

12. D. Lind and B. Marcus, An introduction to symbolic dynamics and coding, Cambridge Univ. Press, 1995. MR 97a:58050

13. R. F. Williams, One-dimensional non-wandering sets, Topology, 6 (1967), 473-487. MR 36:897

14. R. F. Williams, Classification of 1-dimensional attractors, Proc. Symp. Pure Math., 14 (1970), 341-361. MR 42:1134

15. I. Yi, Canonical symbolic dynamics for one-dimensional generalized solenoids, Trans. Amer. Math. Soc., 353 (2001), 3741-3767. MR 2002h:37021

16. I. Yi, Ordered group invariants for one-dimensional spaces, Fund. Math., 170 (2001), 267-286.

17. A. Yu. Zhirov, Hyperbolic attractors of diffeomorphisms of orientable surfaces, Russian Acad. Sci. Sb. Math., 82 (1995), 135-174. MR 95c:58122

Department of Mathematics, University of Maryland, College Park, Maryland 20742

E-mail address: inhyeop@math.umd.edu

Current address: Department of Mathematics and Statistics, University of Victoria, Victoria, British Columbia, Canada V8W 3P4

E-mail address: yih@math.uvic.ca 